\title{
Vector Volume Flow in Arteriovenous Fistulas
}

Hansen, Peter Møller; Heerwagen, Søren; Pedersen, Mads Møller; Rix, Marianne; Lönn, Lars; Nielsen, Michael Bachmann; Olesen, Jacob Bjerring; Pihl, Michael Johannes; Jensen, Jørgen Arendt

Published in:

2013 IEEE International Ultrasonics Symposium Proceedings

Publication date:

2013

Document Version

Early version, also known as pre-print

Link back to DTU Orbit

Citation (APA):

Hansen, P. M., Heerwagen, S., Pedersen, M. M., Rix, M., Lönn, L., Nielsen, M. B., Olesen, J. B., Pihl, M. J., \& Jensen, J. A. (2013). Vector Volume Flow in Arteriovenous Fistulas. In 2013 IEEE International Ultrasonics Symposium Proceedings IEEE.

\section{General rights}

Copyright and moral rights for the publications made accessible in the public portal are retained by the authors and/or other copyright owners and it is a condition of accessing publications that users recognise and abide by the legal requirements associated with these rights.

- Users may download and print one copy of any publication from the public portal for the purpose of private study or research.

- You may not further distribute the material or use it for any profit-making activity or commercial gain

- You may freely distribute the URL identifying the publication in the public portal 


\section{Vector Volume Flow in Arteriovenous Fistulas}

\author{
Peter Møller Hansen, Søren Heerwagen, Mads Møller \\ Pedersen, Marianne Rix, Lars Lönn, Michael \\ Bachmann Nielsen \\ University Hospital of Copenhagen \\ Copenhagen, Denmark
}

\begin{abstract}
The majority of patients with end stage renal disease are in hemodialysis, and therefore dependent on a well functioning vascular access. The arteriovenous fistula is the recommended access and in order to maintain and keep the fistula patent, regular monitoring of the function is necessary. The Ultrasound Dilution Technique is the reference method for volume flow measurement, but it only works in conjunction with the dialysis machine, and use is therefore restricted to dialysis sessions. Volume flow measurement with conventional Doppler ultrasound provides a non invasive, highly accessible solution, but is very challenging due to the angle dependency of the Doppler technique and the anatomy of the fistula. The angle independent vector ultrasound technique Transverse Oscillation provides a new and more intuitive way to measure volume flow in an arteriovenous fistula. In this paper the Transverse Oscillation has been used to measure volume flow directly on four patients' arteriovenous fistulas, and the measurements were compared to subsequent measurements with the Ultrasound Dilution Technique. The results obtained with the Transverse Oscillation deviate $\mathbf{- 3 5 . 1}-\mathbf{1 4 . 9} \%$ from the reference method, and indicates potential for the method.
\end{abstract}

Keywords-arteriovenous fistula; vector ultrasound; transverse oscillation; volume flow, ultrasound dilution, Doppler

\section{INTRODUCTION}

The purpose of this paper is to demonstrate a new clinical use of a novel vector ultrasound technique. Patients suffering from end stage renal disease (ESRD) are completely dependent on dialysis or renal transplant to stay alive. In the U.S. the prevalence rate of ESRD is approximately 1750 per million and the incidence rate is approximately 350 per million per year. $90 \%$ of the ESRD patients are in hemodialysis (HD), which makes it the predominant form of renal replacement therapy [1]. HD is dependent on a well-functioning vascular access with a low rate of complications and sufficient blood flow for the dialysis to be completed in reasonable time. The recommended vascular access for $\mathrm{HD}$ is the arteriovenous fistula (AVF) [2,3]. The AVF is a surgically created anastomosis between an artery and a vein, typically at the wrist, alternatively on the upper arm. The anastomosis of the artery and vein creates a circuit with low resistance leading to highly increased blood flow through the AVF. The increased blood flow leads to a gradual dilation of the vein and thickening of the vessel wall, making it ready for puncture and dialysis after 10 days to 3 months [2]. The hemodialysis vascular access is termed "the lifeline" of ESRD patients, and it

\author{
Jacob Bjerring Olesen, Michael Johannes Pihl, Jørgen \\ Arendt Jensen \\ Center for Fast Ultrasound Imaging \\ Technical University of Denmark \\ Kgs. Lyngby, Denmark
}

is essential to maintain and preserve the functionality of these modified vessels [2,3]. Up to $60 \%$ of ESRD patients with AVF will experience some degree of fistula failure during the first 18 months after the surgical creation of the AVF [4]. The failure is often due to varying degree of stenosis ultimately leading to thrombosis and loss of the AVF [2,3]. Early detection and treatment of stenoses preserves the functionality and patency of the AVF. It is therefore recommended to perform regular surveillance of the AVF function, by measuring the volume flow through the AVF [5,6]. This can be done with several different direct and indirect techniques, among others conventional Doppler ultrasound (DU), magnetic resonance angiography (MRA), and ultrasound dilution technique (UDT). DU has several advantages. It is noninvasive, low-cost, quick to perform, highly mobile, tolerated by all, and provides real-time quantitative velocity estimations along with morphological information about the AVF. The disadvantages to DU are the limited sample volume when performing spectral estimation and the angle dependency, which in combination with the superficial localization and often irregular course of the AVF, makes it difficult to use directly on the AVF [5]. MRA is based on magnetic resonance imaging and provides an accurate, non-invasive estimate of volume flow in an AVF, but is troublesome for the patient, time consuming, expensive, not as available as DU, and therefore not well suited for routine monitoring of the AVF [6]. UDT is the preferred and most widely used technique for measuring volume flow in an AVF, and is considered the reference method [7]. The purpose of this study is to demonstrate how a novel vector ultrasound technique can be used to measure volume flow in an AVF. The new technique Transverse Oscillation (TO) [8] is capable of 2D quantitative angle independent blood flow estimation, and $\mathrm{TO}$ is implemented as "Vector Flow Imaging" (VFI) on several commercial ultrasound scanners from BK Medical.

\section{MATERIALS AND METHODS}

\section{A. Patients}

Four patients with functional AVF's were selected for VFI ultrasound scanning. The patients were recruited upon availability. Three patients have lower arm AVF's and one has an upper arm AVF. All patients entered the study after informed consent. Ethics Committee approval was not necessary for this study, since ultrasound scanning of AVF's is a routine procedure. 


\section{B. Transverse Oscillation}

TO is an angle independent vector technique for velocity estimation with ultrasound proposed by Jensen and Munk [8]. The conventional estimator is used to find the velocity in the axial plane Vz. By subsequently manipulating the receive apodization, the transverse velocity component $\mathrm{Vx}$ is found using the same echo that gave Vz. From these two components, the correct velocity and direction is calculated as a vector [9]. The only change to conventional velocity estimation is the change in receive apodization. TO is FDA-approved and implemented as "Vector Flow Imaging" in several commercial ultrasound scanners from BK Medical. Using VFI the blood flow direction and velocity is illustrated in 2D with color-coded pixels within a color box very similar to conventional color Doppler. To help interpret the flow estimation, the scanner can superimpose arrows real-time on the color-coded pixels. The arrows indicate flow direction and velocity magnitude. TO has previously been demonstrated on healthy subjects [10].

\section{Scan Setup}

A commercial scanner (UltraView 800, BK Medical) was used with a linear transducer (8670, BK Medical). The scanner records VFI-sequences with a frame rate of 15 frames per second and each sequence is by default 15 seconds long. During the ultrasound scan, each patient was in either a hospital bed or a dialysis chair, depending on their normal dialysis routine. Each patient had rested for at least 10 minutes, and blood pressure and heart rate was found to be in habitual predialysis level. Each patient was scanned longitudinally directly on the fistula in order to locate an area with approximately laminar blood flow and regular diameter. (Fig. 1). In the same area the fistula was then scanned transversely to measure two perpendicular diameters of the vessel. (Fig. 2). A small mark was made on the skin next to the transducer, and the transducer was then rotated back in order to record VFIdata longitudinally, making sure the mark on the skin corresponded to the center of the transducer. To ensure that the longitudinal VFI-recording was from the center of the fistula, the transducer was positioned where the fistula had its widest diameter, and the innermost layer of the vessel was visible both at the top and bottom. All scans were performed with a very light touch of the transducer on the skin in order to avoid deformation of the fistula. The scans were performed just prior to insertion of the hemodialysis needles and start of the dialysis. Within the first 30 minutes of dialysis the flow was measured with UDT. From the recorded VFI-data and the geometry of the vessel, volume flow in the fistula was calculated and compared with the UDT measurements.

\section{Ultrasound Dilution Technique}

In this study the UDT measurements were performed using a Transonic HD03 Flow-QC Hemodialysis Monitor (Transonic Systems Inc., Ithaca, NY, USA), and all measurements were made by dialysis nurses with at least five years experience with the Transonic. With UDT volume flow in the AVF is measured by injecting a known amount of indicator (saline) in the bloodstream and subsequently measure the change in indicator concentration as a function of time.

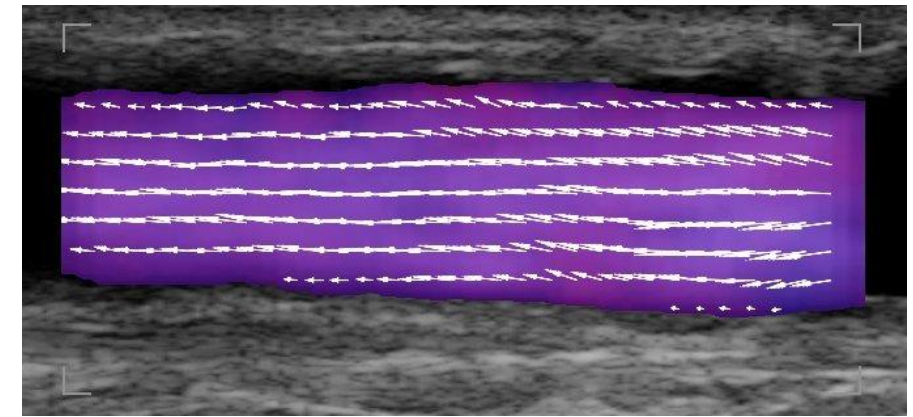

Fig. 1. Longitudinal scan of a regular part of the fistula showing laminar blood flow. Notice the shorter arrows along the wessel walls and the longer arrows toward the middle of the vessel indicating a parabolic flow profile.

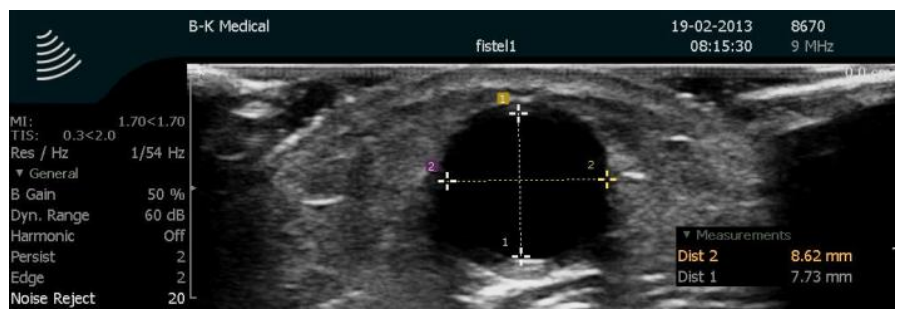

Fig. 2. Transversal scanning of the fistula with measurements of diameters.

This is done via the dialysis machine, where the dialysis blood lines have been reversed for the flow measurement. Between two consecutive measurements with the Transonic there is an expected variation of $100 \mathrm{ml} / \mathrm{min}$ in flows lower than 1000 $\mathrm{ml} / \mathrm{min}$ and $10 \%$ expected variation in flows larger than 1000 $\mathrm{ml} / \mathrm{min}$. If two consecutive measurements are within these limits, the final result is the average of the two [11]. The disadvantages of UDT are the need for dialysis to be up and running and the reversal of dialysis blood lines before an estimation of flow is obtainable. This process takes time from the dialysis and is uncomfortable for both patient and dialysis nurse.

\section{E. Calculation of flow from VFI}

From each fistula 15 seconds of longitudinal VFI-scanning was recorded. If the patients arm moved during the recording, that specific sequence was deleted. The sequences were analyzed off-line using MATLAB (MathWorks, Natick, MA, USA). From each sequence the peak velocities in the center of the fistula during systole and diastole were identified from the VFI data. Because of the high number of still frames which should be processed by the computer and examined individually and manually, the velocities were only obtained from two consecutive heart cycles. With a heart rate of 60 beats per minute, two cycles correspond to 30 frames. The final peak velocity estimates (systolic and diastolic) were then calculated as an average of the two heart cycles. The velocities were identified from the part of the VFI data corresponding to the center of the transducer. Assuming laminar flow and a parabolic flow profile, the average flow velocity across a vessel is given by

$$
\operatorname{Vavg}=\mathrm{Vmax} / 2
$$


The cross sectional area of each fistula was calculated as an ellipse by

$$
\mathrm{A}=\pi * \mathrm{r} 1 *_{\mathrm{r} 2}
$$

and volume flow (VF) at a given point in the cardiac cycle is given by

$$
\mathrm{VF}=\mathrm{Vavg} * \mathrm{~A}
$$

Volume flow was calculated for systole and diastole individually. The volume flow for the entire cardiac cycle was then calculated as a weighted average from the systolic and diastolic values. When a person is in rest the systole accounts for approximately one-third of the cardiac cycle and the diastole accounts for two-thirds [12]. Volume flow for the entire cardiac cycle in each fistula was therefore calculated using

$$
\text { VFtotal }=1 / 3 * \text { VFsystole }+2 / 3 * \text { VFdiastole }
$$

\section{RESULTS}

For each of the four patients volume flow was calculated from two consecutive heart cycles recorded in one VFI sequence. All UDT measurements were made according to the manufacturers guidelines [11]. The deviation is calculated relative to the reference method Transonic. See results in table 1.

\section{DISCUSSION}

The calculated VFI volume flows indicate that it is possible to obtain a reasonable volume flow measurement directly from an AV-fistula with a conventional ultrasound scanner. When comparing the flow results from VFI and UDT, it is important to keep the expected variations of the Transonic in mind. The angle independence of VFI makes flow estimation more intuitive and less operator dependent compared to conventional Doppler. It is now possible to evaluate the flow conditions in the fistula at all angles of insonation, and at the same time obtain quantitative information about velocity and direction of the flow from anywhere within the color box. Any kind of disturbed flow is visualized along with the flow profile. The usual assumptions of laminar flow and a parabolic flow profile needed for conventional Doppler estimation are therefore not an issue. With VFI the operator can immediately see whether the flow is approximately laminar or disturbed, where in the scan plane the highest flow velocities are found, and is not restricted by size or placement of the Doppler sample volume for spectral measurements. The angle independence is furthermore an advantage when holding the transducer against the skin. Just the weight of the transducer is enough to compress and deform the fistula, thereby creating disturbed and unpredictable flow, and it is therefore a challenge to obtain the recommended angle of insonation less than $60^{\circ}$ for conventional spectral estimation [13]. Using VFI the operator can focus on scanning with a very light touch without deforming the fistula. One of the limitations of TO compared to UDT is the 2D visualization of the flow. That makes the VFI measurement most suited for calculation of volume flow in a
TABLE I.

\begin{tabular}{|c|c|c|c|}
\hline Patient & $\begin{array}{c}\text { Vector flow } \\
\mathbf{m L} / \mathbf{m i n}\end{array}$ & $\begin{array}{c}\text { Transonic } \\
\mathbf{m L} / \mathbf{m i n}\end{array}$ & $\begin{array}{c}\text { Deviation } \\
\%\end{array}$ \\
\hline 1 & 824 & 717 & 14.9 \\
\hline 2 & 1035 & 1595 & -35.1 \\
\hline 3 & 411 & 555 & -25.9 \\
\hline 4 & 431 & 430 & 0 \\
\hline
\end{tabular}

circular blood vessel, since it is only possible to obtain VFI data from the longitudinal scan plane going from top to bottom of the vessel. Since the cross sectional area of the fistulas in all four cases is elliptical with the longest diameter going from side to side, the volume flow must be expected to be higher than what can be calculated from the VFI recording from the shorter diameter only. The maximum difference between the two diameters of the fistula was found to be $12 \%$ in patient 1 . In the remaining three patients the difference was less than 7 $\%$, corresponding to the same differences in flow calculation. Another limitation of TO compared to UDT, is that volume flow in this study is only calculated during two cardiac cycles. The Transonic measures flow during several cardiac cycles, and a better estimate of the average flow is therefore obtained. Further work for optimizing and automating the calculation of volume flow from VFI is taking place among the authors.

\section{CONCLUSION}

This study demonstrates the first examples of a commercial vector ultrasound system used for volume flow measurements in AV-fistulas. Besides a reasonable volume flow VFI provides further information about flow phenomena in the fistulas, not detected by conventional Doppler. The angle independence of VFI makes the flow evaluation and estimation more intuitive and less operator dependent, leading to potential increased use of ultrasound for these types of examinations. Any ultrasound scanner provides morphological information from a fistula, and the combination with VFI can make this technique an important tool for diagnostic examination and monitoring of AV-fistulas and other blood vessels.

\section{REFERENCES}

[1] kidney.niddk.nih.gov/kudiseases/pubs/kustats/

[2] K Konner, B Nonnast-Daniel,and E Ritz, "The Arteriovenous Fistula," J Am Soc Nephrol 14: pp. 1669-1680, 2003.

[3] JA Bittl, "Catheter Interventions for Hemodialysis Fistulas and Grafts,' JACC: Cardiovascular Interventions, vol. 3, No. 1: pp. 1-11, 2010.

[4] HJT Huijbregts, ML Bots, CHA Wittens, YC Schrama, FL Moll, and PJ Blankestijn, "Hemodialysis Arteriovenous Fistula Patency Revisited: Results of a Prospective, Multicenter Initiative," Clin J Am Soc Nephrol 3: pp. 714-719, 2008.

[5] P Wiese, and B Nonnast-Daniel, "Colour Doppler ultrasound in dialysis access,” Nephrol Dial Transplant 2004; 19: pp. 1956-1963.

[6] WL Whittier, "Surveillance of Hemodialysis Vascular Access," Seminars in interventional radiology; volume 26; number 2, pp. 130138, 2009.

[7] E Wijnen, S Essers, G van Meijel, JP Kooman, J Tordoir et al, "Comparison Between Two On-line Reversed Line Position 
Hemodialysis Vascular Access Flow Measurement Techniques: Saline Dilution and Thermodilution," ASAIO J 2006; 52: pp. 410-415.

[8] J. A. Jensen and P. Munk, "A new method for estimation of velocity vectors," IEEE Trans Ultrason Ferroelec Freq Contr, vol. 45, pp. 837 851, 1998.

[9] J. A. Jensen, "A new estimator for vector velocity estimation", IEEE Trans Ultrason Ferroelec Freq Contr, vol. 48, pp. 886-894, 2001.

[10] PM Hansen, MM Pedersen, KL Hansen, MB Nielsen, and JA Jensen, "New technology - demonstration of a vector velocity technique," Ultraschall in Med 32; pp. 213-215, 2011.
[11] http://www.transonic.com/inventory/view/18031

[12] T Bombardini, V Gemignani, E Bianchini, L Venneri, C Petersen, et al, "Diastolic time - frequency relation in the stress echo lab: filling timing and flow at different heart rates", Cardiovascular Ultrasound 2008, 6:15, 20 pages.

[13] V Teodorescu, S Gustavson, and H Schanzer, "Duplex Ultrasound Evaluation of Hemodialysis Access: A Detailed Protocol,” Int J Nephrol, vol. 2012, 7 pages. 